\title{
论 文
}

\section{典型亚热带森林生态系统WUE对物候变化的响应 以浙江省森林生态系统为例}

郭丰生 ${ }^{1,2}$, 金佳至金 ${ }^{1, *}$, 雍斌 1,2, 王颖 ${ }^{3}$, 江洪 $^{4}$

1. 水文水资源与水利工程科学国家重点实验室, 南京 210098 ;

2. 河海大学地球科学与工程学院, 南京 211100 ;

3. 三江学院文化产业与旅游管理学院, 南京 210012;

4. 南京大学国际地球系统科学研究所, 南京 210046

* 通讯作者, E-mail: jiaxinking@hhu.edu.cn

收稿日期：2018-10-02; 收修改稿日期：2019-04-08; 接受日期：2019-04-15; 网络版发表日期：2019-05-17

国家重点研发计划项目(编号: 2018YFA0605402)、国家自然科学基金项目(批准号: 41601442和41807173)和中央高校基本科研业务费项目(编 号: 2017B06814)资助

摘要 生态系统水分利用效率(WUE)是衡量生态系统碳水耦合循环程度的重要指标. 已有研究表明, 非生物和生 物因子都会对森林生态系统WUE产生显著的影响，然而，在气候变化背景下对于WUE对物候响应的认识还不够 深入. 文章利用遥感数据，以浙江省为研究区，分析2000 2014年季节性WUE对物候的敏感性和响应模式的空间 变异，并探讨这两者随不同气候梯度的变化规律和成因。WUE与物候年际之间的偏相关分析表明，春季WUE对 $\mathrm{SOS}$ 的敏感性为负，主要是因为随着生长季开始(SOS)的提前，总初级生产力(GPP)的增加幅度大于蒸散发(ET); 夏季WUE对SOS的正敏感性主要跟水分限制及植物生态对策有关; 秋季WUE对生长季结束(EOS)的正敏感性主 要是由于随着EOS延后, GPP增加而ET变化不大或者减小所致. 在空间上, 春季WUE对SOS的敏感性随辐射梯度 显著减弱, 这可能与高辐射地区较强的土壤蒸发有关; 夏季WUE对SOS的敏感性与降水表现出正相关关系, 与温 度表现为负相关关系, 这可能归因于高温导致的水分胁迫和GPP对SOS延迟的补偿作用; 秋季WUE与EOS的敏感 性随辐射和降水梯度显著增强，这可能跟辐射和降水的增加为光合作用提供更多的能量和水分有关.

关键词水分利用效率, 总初级生产力, 蒸散发, 物候, 气候梯度, 森林

\section{1 引言}

作为地球表层系统能量转换和物质循环的核心, 陆地生态系统碳水耦合循环是生态系统功能研究的关 键科学问题(Liu等, 2018; Li等，2019). 水分利用效率 (water use efficiency, WUE)是衡量碳水耦合循环程度 的综合性指标(Le Houerou, 1984; Huang等, 2017), 一 直受到植物生理学和生态学等领域广泛的关注(Reichstein等, 2010; Huang等, 2015; Guerrieri等, 2016; Liu等, 2019). 不同的研究尺度对应不同的WUE定义(Beer等, 
2009; Ponton等, 2010; Zhou等, 2014), 在生态系统尺度 上, WUE被定义为总初级生产力 (gross primary productivity, GPP)与蒸散发(evapotranspiration, ET)的比值, 表 示植物每消耗单位重量的水所产生的干物质的量 (Keenan等, 2013; Tang等, 2015), 是对生态系统植被组 成和蒸发的综合响应体现(Law等，2002; Huang等, 2017). 当今，全球气候发生显著变化(Wolf等，2016), 揭示生态系统WUE的时空变异机制有助于预测并应 对未来气候变化对碳循环和水循环的影响(Yang等, 2014).

物候是指自然界中的生物受气候和其他环境因素 的影响而出现的现象, 是生物周期性的生长变化 (Morisette等, 2009). 通常而言, 植物物候是指植物生 长周期中某一事件发生的时间节点或不同状态之间的 转折点, 如落叶植物的发芽、常绿植物叶片从休眠状 态进入光合作用状态的转折点等. 从植物个体到生态 系统各个尺度, 众多过程都由物候直接或间接调控, 特别是与碳(光合与呼吸)、水(蒸腾与蒸发)循环相关 的过程(Reed等, 2009).

现有研究表明, 物候对气候变化非常敏感, 物候变 化趋势与生长季的气候变化趋势相吻合(Piao等, 2015; Ge等, 2016; Dai等, 2017; Richardson等, 2013), 因此气 候变化将导致物候发生变化(Wang等, 2015, 2017), 进 而植被生理活性的时序进程与强度也将会发生变化. 例如, 在早春年份, 发芽或叶片复苏提前将会促进植被 较早进入生长阶段, 增加生长季初期的GPP(Richardson等, 2010; Jin等, 2017b), 同时叶面积增加也会增加 植物的蒸腾量(Beer等, 2009) 以及降低土壤蒸发 (McPherson, 2007; Shen等, 2015). 这些变化最终将导 致生态系统WUE的异常波动. 但迄今为止, 气候变化 背景下WUE的物候调控机制认识还不够深入.

考虑到未来物候和气候变化对生态系统WUE的 重要作用, 本文以浙江省为研究区, 针对典型亚热带森 林生态系统, 利用遥感数据监测该地区森林生态系统 WUE和物候, 分析森林生态系统WUE对物候响应敏 感性及响应模式, 阐明季节性WUE对物候的敏感性和 响应模式随不同气候梯度的变化特征, 探讨不同气候 梯度下森林WUE对物候季节响应差异的成因. 实现以 上目标, 将有助于加深对气候变化下森林生态系统碳 水耦合循环的生物调控机制的认识, 提高亚热带森林 生态系统碳-水循环变化预测的能力, 同时对森林生态
系统WUE测定与预测的模型参数确定提供科学依据.

\section{2 研究区与数据}

\section{1 研究区}

浙江省地处中国东南沿海、长江三角洲南翼, 纬 度约为 $27^{\circ} \mathrm{N} \sim 31^{\circ} \mathrm{N}$, 经度约为 $118^{\circ} \mathrm{E} \sim 123^{\circ} \mathrm{E}$ (图1). 浙江 省属于典型亚热带季风气候, 四季分明, 光照充足, 热 量丰富, 年均气温为 $15 \sim 18^{\circ} \mathrm{C}$, 日均时照数为 $4.6 \sim 5.8 \mathrm{~h}$. 近60年来, 浙江省气温主要呈现上升、降雨主要呈现 下降的变化特点, 气候趋于“热干化”. 浙江省森林植 被属于中亚热带常绿落叶阔叶混交林, 森林资源丰富, 常见植被有常绿阔叶林、落叶阔叶林、常绿阔叶落叶 混交林. 考虑到当地气候变化以及丰富的自然资源, 研 究浙江省森林生态系统的物候和WUE相互作用关系, 从经济角度和生态角度来说都具有重大意义. 另外由 于浙江省森林生态系统属于典型亚热带森林生态系 统, 因此本研究也具有一定的可推广性.

\subsection{MODIS GPP和ET数据}

在生态系统WUE研究中, 遥感数据具有时间长、 范围广等优势(Tang等, 2015; Sun等, 2016). 本研究采 用2000 2014年空间分辨率为 $0.05^{\circ}$ 的MODIS GPP (MOD17)和ET(MOD16)数据(http://www.ntsg.umt.edu/), GPP单位为 $\mathrm{g} \mathrm{C} \mathrm{m}^{-2}$, ET的单位为 $\mathrm{mm}$, 分别利用光能利 用率模型和Penman-Monteith方法从卫星植被信息数 据和气象数据反演获得, 数据详情参见Running等 (2004)、Zhao等(2005)和 Mu等(2011). 本研究以GPP和 ET月均值数据为基础, 计算GPP和ET季节均值, 并计 算各季节的GPP和ET的比值获得森林生态系统季节 性WUE. 本研究分别将3 5月、6 8月和9 11月定义为 春季、夏季和秋季.

\section{3 气候数据}

本研究所使用的气候因子包括近地面温度 $\left({ }^{\circ} \mathrm{C}\right)$, 降水率 $\left(\mathrm{mm} \mathrm{h}^{-1}\right)$ 和向下短波太阳辐射 $\left(\mathrm{MJ} \mathrm{m}^{-2} \mathrm{day}^{-1}\right)$. 气候数据来源于中国区域高时空分辨率地面气象要素 驱动数据集, 其时间分辨率为 $3 \mathrm{~h}$, 空间分辨率为 $0.1^{\circ}$ (westdc.westgis.ac.cn/data/). 中国区域地面气象要素数 据集是中国科学院青藏高原研究所开发的一套近地面 气象与环境要素再分析数据集. 该数据集是以国际上 


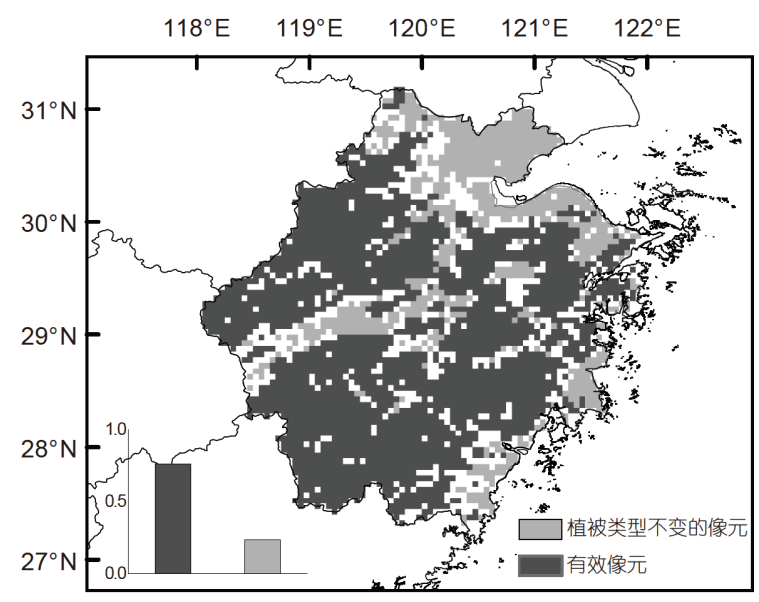

图 1 研究区植被分布图

深灰色像元表示2001 2012年均为森林覆盖的像元, 浅灰色像元为 最终篮选得到的有效像元

现有的Princeton再分析资料、GLDAS资料、GEWEX-SRB辐射资料，以及TRMM降水资料为背景场， 融合了中国气象局常规气象观测数据制作而成(Chen 等, 2011). 为与其他数据空间分辨率相匹配, 本研究通 过空间插值的方法将气候数据的空间分辨率由 $0.1^{\circ}$ 插 值成 $0.05^{\circ}$.

\section{4 土地覆盖数据}

本研究所用到的土地覆盖类型数据来源于 MODIS土地覆盖类型产品(MCD12C1), 该产品的空间 分辨率为 $0.05^{\circ}$, 提供了土地覆盖类型评估、百分比分 布和质量控制信息. MODIS土地覆盖类型产品包括从 每年Terra星数据中提取的土地覆盖特征不同分类方 案的数据分类产品. 本研究选取的土地覆盖类型为 IGBP(International Geosphere Biosphere Programme) 定义的 17 类, 包括 11 类自然植被分类、3类土地利用和 土地镶嵌、 3 类无植生土地分类. 数据分类来自监督决 策树分类方法, 分类精度达 $75 \%$. 为降低土地覆盖类型 变化导致的WUE变化，本研究利用2001 2012年浙江 省土地覆盖数据提取森林生态系统.

\section{3 方法}

\section{1 数据预处理}

由于云、雪以及降雨等天气的影响和各种误差, 部分数据存在着质量问题, 造成结果的不确定性. 本研
究按照以下条件篮选研究区内有效像元: (1) 对于土地 覆盖数据, 保留12年均为森林覆盖的像元; (2) 选择夏 季活跃型森林生态系统, 即夏季 GPP均值在一年中最 高，冬季(12 2月)GPP均值在一年中最低; (3) 每年有 效的GPP数据记录应该大于总数据记录的 $80 \%$. 这样 有助于本研究后续结果分析更具准确性与科学性.

\section{2 动态阈值法}

不同于传统意义上的植被物候现象，在利用基于 卫星遥感数据进行物候提取时, 所得的“物候”表征从 一种状态到另一种状态的转换日期, 即状态切换时间. 利用遥感卫星数据进行区域尺度上的植被物候提取方 法较多, 考虑到动态阈值法能够不受土地覆盖类型的 影响, 得到比较具有针对性的物候指标, 所以在本研 究中采用动态阈值法进行物候点的提取. 本研究选取 以 GPP为基础的光合作用周期中春、秋两季状态的转 换日期, 即生长季开始(SOS)和结束(EOS). 利用每个有 效像元的分辨率为 8 天的 GPP数据作为物候点提取的 数据源, 通过MATLAB对GPP数据进行线性插值计算, 得到GPP日数据; 通过SSA滤波对所得数据进行平滑 (Keenan等, 2014). 由于此次所选研究区为典型亚热带 森林生态系统，其中的亚热带常绿阔叶林年内季相变 化不明显, 因此本研究利用 8 天分辨率的GPP数据以及 $10 \%$ 的动态阈值提取生长季的开始点和结束点, 得到 植物的物候. 该物候表征了植物碳同化能力的转变, 以此代替“归一化植被指数(NDVI)”数据所提取的植 被 “绿度”物候，用以捕捉亚热带常绿阔叶林的物候变 化(Wu等, 2012, 2013).

\section{3 统计分析方法}

本研究采用线性回归分析法分别分析研究区内季 节性WUE、物候的年际变化趋势; 利用偏相关分析法 在控制气候因子(温度、降水和辐射)后分析了季节 WUE与物候期之间的偏相关性; 根据WUE、GPP和 ET与物候之间的偏相关性进一步识别WUE对物候的 六种响应模式，并且按像元气候多年均值排序建立气 候梯度, 进行WUE对物候敏感性和响应模式的统计 (Jin等，2017a); 为了降低异常值的干扰，采用Theil Sen's slope和Mann-Kendall趋势检验法分析WUE变化 与物候变化、WUE敏感性与气候梯度间关系(Wang 等, 2018). 


\section{4 结果}

\subsection{WUE与物候的年际变化}

图2展示了2000 2014年研究区森林生态系统 WUE季节均值和生长季开始(SOS)、结束(EOS)日期
年际变化的空间分布. 在春季，森林WUE整体表现为 逐年上升的趋势，平均每年增加 $(0.0024 \pm 0.0038) \mathrm{g} \mathrm{C}$ $\mathrm{m}^{-2} \mathrm{~mm}^{-1}$, 其中, $9.79 \%$ 的有效像元线性存在显著变化 趋势， $75.48 \%$ 的有效像元呈现逐年上升趋势，增幅为 (0.0059 \pm 0.0028$) \mathrm{g} \mathrm{C} \mathrm{m}^{-2} \mathrm{~mm}^{-1} \mathrm{a}^{-1} ; 24.52 \%$ 的有效像元

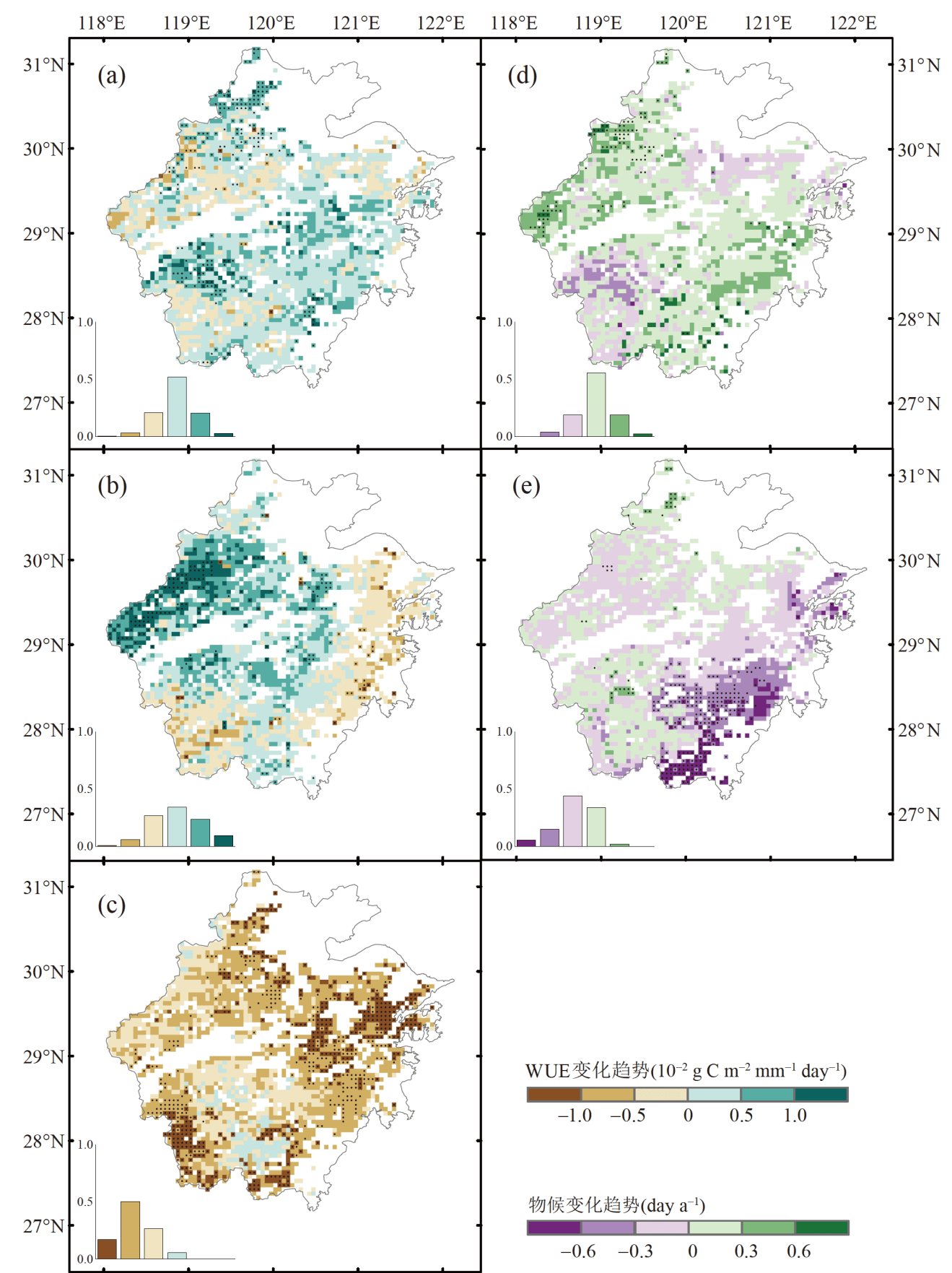

图 $22000 \sim 2014$ 年水分利用效率WUE $((a) \sim(c))$ 和生长季开始点 $\operatorname{SOS}(\mathrm{d})$ 及结束点 $\operatorname{EOS}(\mathrm{e})$ 线性趋势的空间分布 WUE分别为春季、夏季、秋季的WUE线性趋势. 白色区域为无效像元, 带点像元表示该像元线性趋势显著 
呈现逐年下降趋势，降幅为 $(0.0024 \pm 0.0023) \mathrm{g} \mathrm{C} \mathrm{m}^{-2}$ $\mathrm{mm}^{-1} \mathrm{a}^{-1}$. 具体而言, 下降的区域主要位于研究区 $29^{\circ} \mathrm{N} \sim 30^{\circ} \mathrm{N}$ 和西北部的森林生态系统, 其他森林生态 系统WUE均在逐年增加(图2a). 在夏季, 研究区森林 生态系统的WUE整体同样表现出上升的趋势， $9.31 \%$ 的有效像元线性趋势达到显著, 但夏季WUE上升的区 域占比(有效像元的 $66.91 \%$ )比春季有所减小. 然而, 夏季WUE整体表现出来的上升趋势强度 $((0.0028$ $\pm 0.0058) \mathrm{g} \mathrm{C} \mathrm{m}^{-2} \mathrm{~mm}^{-1}$ )大于春季(图2b). 与春季和夏季 不同，秋季的WUE表现出大范围的下降趋势，且 $27.67 \%$ 的有效像元变化趋势达到显著性水平，平均每 年下降 $(0.0066 \pm 0.004) \mathrm{g} \mathrm{C} \mathrm{m}^{-2} \mathrm{~mm}^{-1}$, 下降强度大于春 季和夏季WUE的上升强度, 其中 $94.36 \%$ 的有效像元表 现逐年下降趋势(图2c).

在整个研究区内, 春季SOS年际回归系数分布在 $-0.66 \sim 1.17$ day a $^{-1}$, 其中 $76.92 \%$ 区域表现出SOS延迟 的现象, 平均每十年生长季开始点延迟 $(2.3 \pm 1.7)$ 天, 剩 余23.08\%区域存在SOS提前的现象，平均每十年SOS

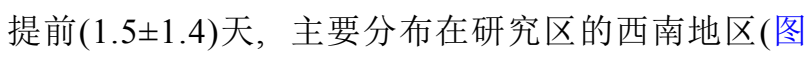
$2 \mathrm{~d})$, 但只有 $4.68 \%$ 的有效像元趋势通过显著性检验. 从图 $2 \mathrm{e}$ 可以看出, 秋季 $\mathrm{EOS}$ 年际回归系数在 $-1.01 \sim 0.48 \mathrm{day} \mathrm{a}^{-1}, 11.54 \%$ 的有效像元变化趋势达到 显著性水平, 并且EOS的提前和延迟在整个研究区范 围内均有分布, $35.71 \%$ 的区域存在着EOS延迟的现象, 平均每十年延迟 $(1.0 \pm 2.1)$ 天, 这可能与 $S O S$ 的延迟有关 (Fu等, 2014; Keenan和Richardson, 2015; Liu等, 2016); $64.29 \%$ 的区域存在EOS提前的现象，平均每十年生长 季提前(2.4 \pm 0.9$)$ 天, 主要集中位于研究区的东部区域.

图3展示了空间上，不同物候变化趋势下各季节 WUE趋势的变化特征. 图中横轴为有效像元物候的年 际变化趋势, 纵轴为对应的季节WUE的年际变化趋势. 图中每个点代表了按横轴排序后从低值起邻近的 30 个 点的平均值. 从空间上看, 春季WUE年际变化趋势与 $\mathrm{SOS}$ 年际变化趋势呈负相关 (Sen's slope $=-0.004$, $p<0.01$ ), 即 $\mathrm{SOS}$ 提前幅度越大的森林生态系统, 其 WUE增加的幅度越强, SOS延后幅度越大的地区，其 WUE增加的幅度越弱，甚至出现逐年降低的趋势(图 3a). 不同于春季，夏季WUE年际变化趋势与SOS年际 变化趋势呈现空间正相关 (Sen's slope $=0.0039$, $p<0.01)$, 即 $\mathrm{SOS}$ 延后幅度越大的森林生态系统, 其 WUE增加的幅度越强, SOS提前幅度越大的地区, 其
WUE年际变化趋势越趋向于 0 值, 即WUE趋于稳定状 态(图3b). 秋季的结果(图3c)显示, 无论生长季结束日 期延迟或者提前, WUE整体都表现为下降趋势, 且空 间上秋季WUE和EOS的年际变化趋势呈现正相关, 即 在EOS延后幅度越大的森林生态系统, 其WUE减少的 幅度越弱, 在EOS提前幅度越大的森林生态系统, 其 WUE减少的幅度越大. 换而言之, 生长季延后在一定 程度上会减弱秋季森林生态系统WUE的下降强度.

\subsection{WUE与物候的相关关系}

以温度、降水率、辐射三个环境因子为控制变 量, 得到各季节WUE与物候之间的偏相关系数. 图4展 示了各季节WUE与物候之间的偏相关系数的空间分 布状况. 春季WUE与SOS整体上表现为负相关关系 $(r=$ $-0.2 \pm 0.2)$, 其中 $20.94 \%$ 的有效像元显著相关. 其中 $81.42 \%$ 的有效像元表现为负相关关系, 平均Pearson相 关系数为 $-0.3 \pm 0.2$, 而表现为正相关关系的有效像元 偏相关系数基本上小于 0.3 , 主要分布在研究区的西北 部, 平均Pearson相关系数仅为 $0.1 \pm 0.1$ (图 $4 a$ ). 与春季相 反, 夏季 WUE与春季物候正相关关系占主导地位 $(r=0.3 \pm 0.3), 34.13 \%$ 的有效像元相关性表现为显著. 其 中 $82.43 \%$ 的有效像元表现为正相关关系, 平均Pearson 相关系数为 $0.4 \pm 0.2,17.57 \%$ 的有效像元表现为负相关 关系, 平均Pearson系数为 $-0.3 \pm 0.2$, 主要集中分布在研 究区的东北部地区(图4b). 对于秋季而言, WUE与EOS 普遍呈现正相关性 $(r=0.3 \pm 0.2), \quad 34.18 \%$ 的有效像元相 关性达到显著性. 其中， $85.58 \%$ 的有效像元表现为正 相关关系, 平均Pearson相关系数为 $0.4 \pm 0.2,14.42 \%$ 的 有效像元表现为负相关关系, 平均Pearson系数为 -0.1 \pm 0.1 , 分布比较零散且相关性不高(图4c).

为探究气候变化对WUE生物调控作用的潜在影 响, 本研究进一步分析了季节性WUE对物候变化敏感 性随气候梯度(温度、降水率、辐射的多年均值)的空 间变异(图5). 为降低异常值干扰, 采用Theil Sen's slope 和Mann-Kendall趋势检验法分析WUE对物候的敏感 性与气候梯度间的关系. 结果表明, 在春季, WUE与 $\operatorname{SOS}$ 的负相关性随辐射梯度变化显著, 且随辐射的增 强而减弱(Sen's slope $=0.0059, p=0.019$ ); 随温度和降 水率梯度, WUE对物候的敏感性无显著变化 $(p>0.05)$. 夏季WUE与SOS的正相关性沿降水率和温度梯度变 化显著. 具体而言, 随降水率的增加, 夏季WUE与SOS 

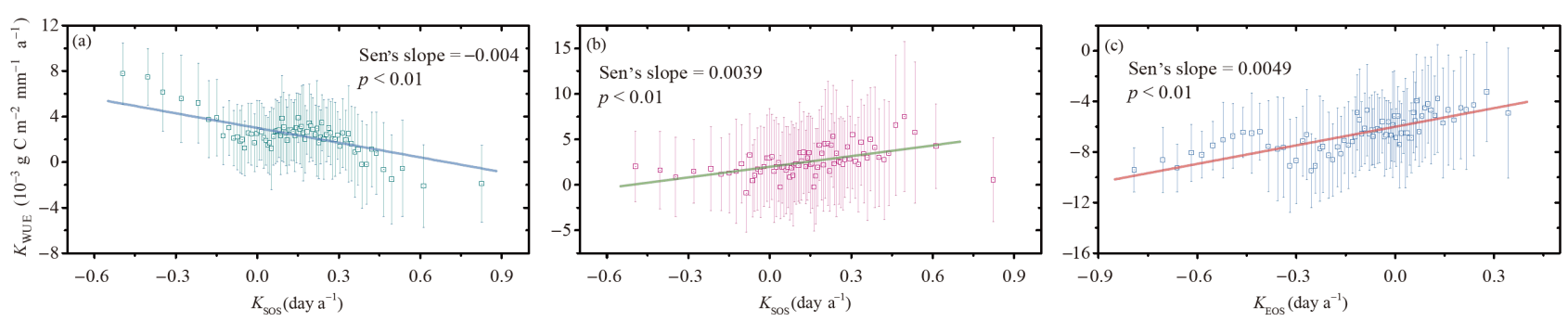

图 3 不同物候变化趋势下季节性水分利用效率WUE年际趋势的变化特征

横轴为有效像元物候的年际变化趋势, 纵轴为对应的季节WUE的年际变化趋势. 每个点代表了按横轴排序后从低值起邻近的 30 个点的平均 值. (a) ( c) 分别对应春季、夏季、秋季

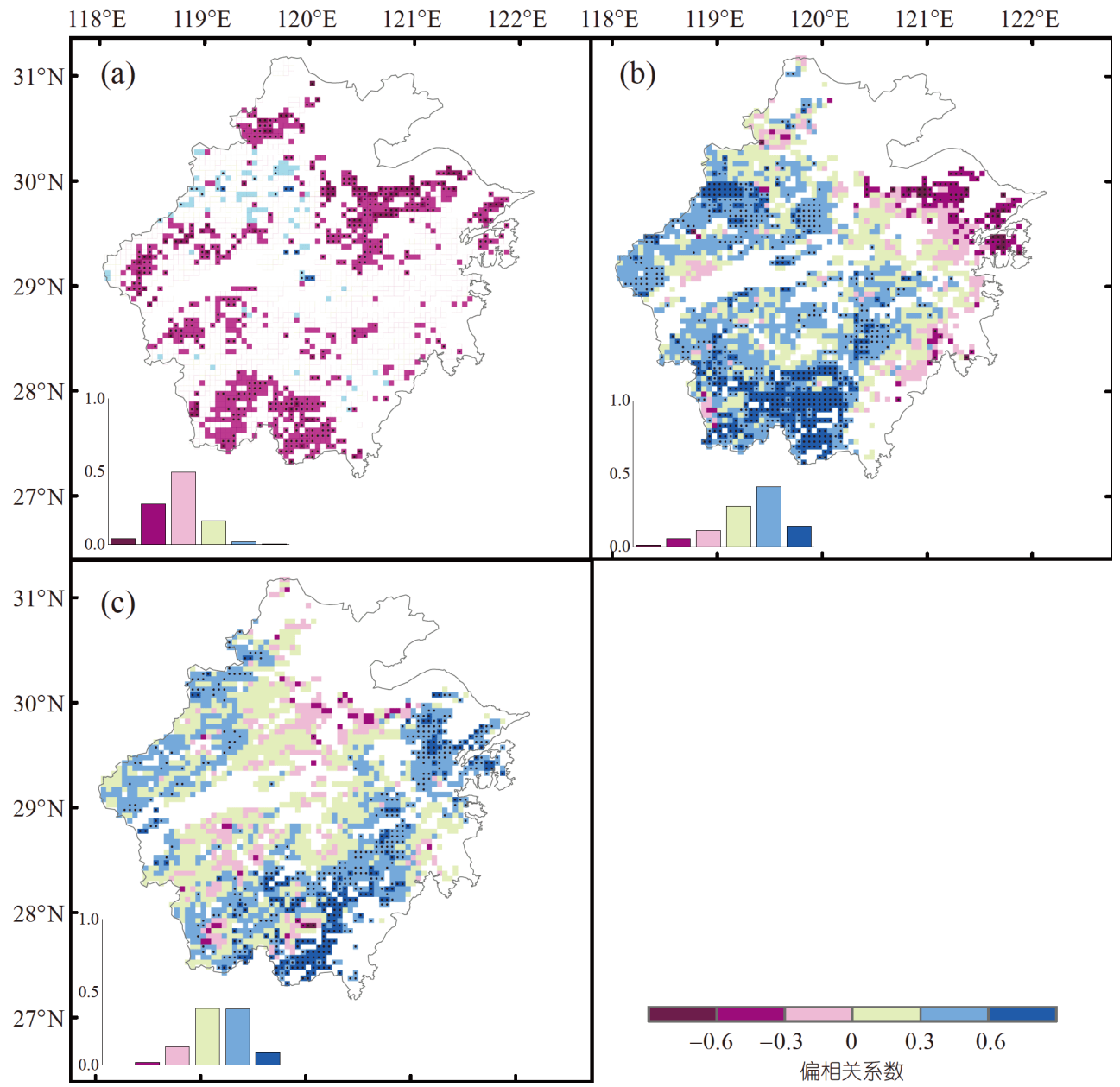

图 4 季节性水分利用效率WUE和物候期(SOS、EOS)间偏相关系数的空间分布

偏相关系数在控制气候因子(温度、降水和辐射)后计算得到. (a) ( c) 分别对应春季、夏季、秋季. 白色区域为无效像元, 带点像元表示该像元 相关性显著

的正相关性增强(Sen's slope $=1.2528, p<0.01$ ), 即夏季 降水越高的区域, 生长季开始期延后对夏季WUE的促 进作用越强; 随温度的升高, 夏季WUE与SOS的正相
关性减弱 $(\mathrm{Sen}$ 's slope $=1.2528, p<0.01)$, 即夏季温度越 高的区域, 生长季延迟对夏季WUE的促进作用越弱; 随辐射梯度, WUE对物候的敏感性无显著变化 

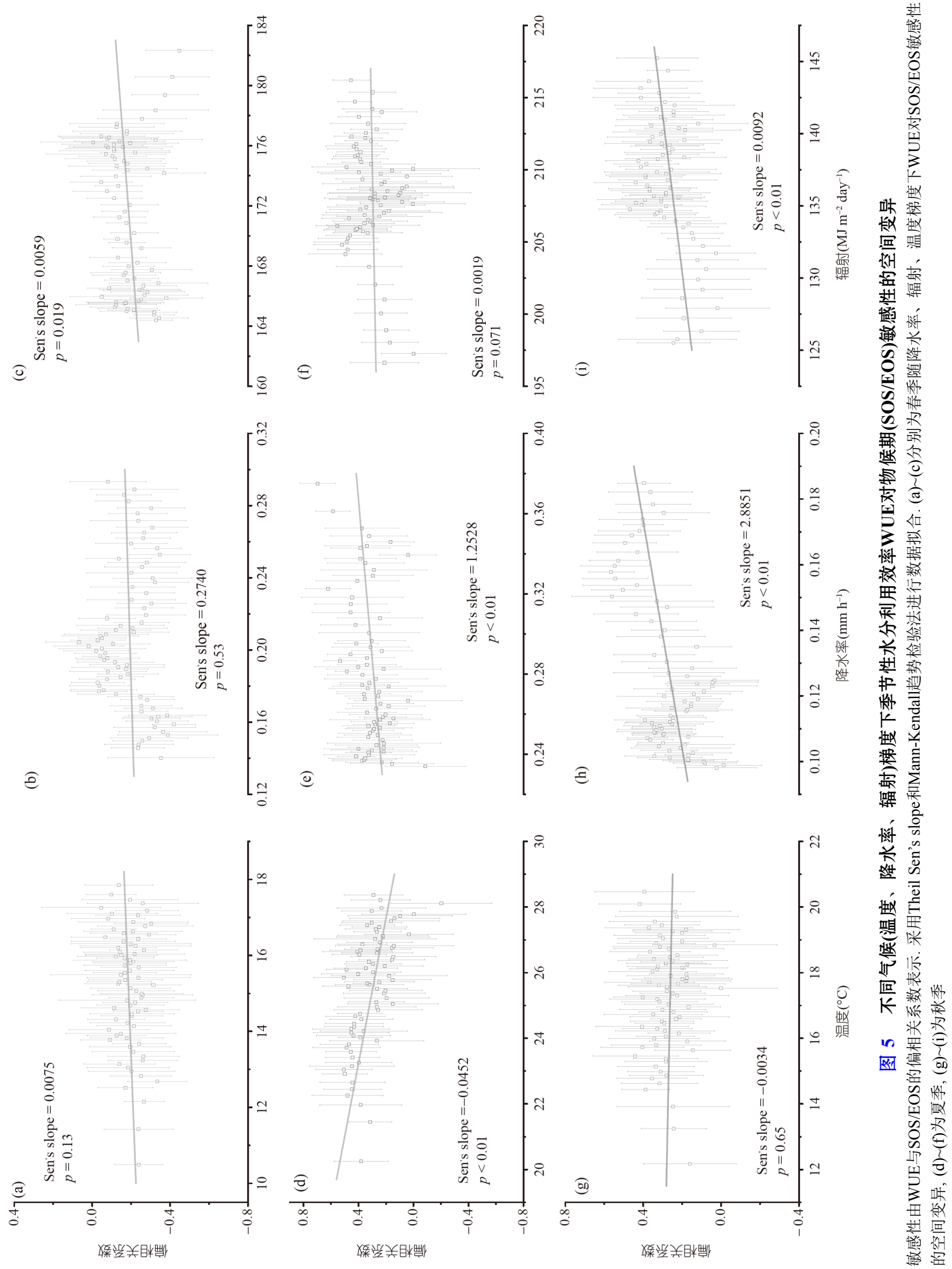
$(p>0.05)$. 秋季的结果显示, 其WUE与EOS的正相关性 沿降水率和辐射梯度变化显著. 由于降水增加使得原 本缺水的秋季土壤变得湿润, 植被生理活性增强, 从 而WUE与EOS的正相关性随降水的增加而显著增强 (Sen's slope $=2.8851, p<0.01$ ), 即秋季高降水区生长季 结束期延后对WUE的促进作用比低降水区更强; 与沿 降水梯度结果类似, 辐射的增强, 为植被光合作用带来 更多的能量, GPP增加, 从而WUE与EOS的正相关性也 表现出增加的趋势, 但增加趋势较小 (Sen's slope= $0.0092, p<0.01)$. 结果表明, 秋季高辐射区域, 生长季 延后对其WUE的促进更为明显; 随温度梯度, 拟合效 果不显著 $(p>0.05)$.

\subsection{WUE对物候的响应模式}

WUE对物候响应表征了 GPP和ET对物候响应的 耦合, 本研究通过研究区春、夏、秋季各季节森林生 态系统WUE、GPP、ET与物候点的偏相关性, 识别了 WUE对物候变化的六种响应模式, 空间结果如图6 所示.

对于春季WUE的响应而言, 模式VI占主导地位, 占有效像元的 $41.1 \%$ (图6a), 表明春季WUE与 SOS的负 相关性主要是因为随着SOS提前, 植被提前开始光合 作用, 增加春季GPP, 叶面积的增大同时也增大了春季 $\mathrm{ET}$, 但此时 GPP增加幅度大于ET. 夏季模式 I 和模 式 II 分别占有效像元的 $46.7 \%$ 和 $32.6 \%$, 表明夏季WUE 与SOS的正相关性主要由以下两点原因造成: (1) SOS 提前导致土壤水分含量较低, 夏季GPP和ET都减少, 但 GPP减少幅度大于ET; (2) 夏季GPP随着SOS提前而降 低, 而夏季ET仍在增加. 这两种情况都有可能造成夏 季WUE随着SOS提前而降低. 对于秋季而言, 同样是 模式 I 和模式 II 占主导地位, 分别占有效像元的 49.7\%和 28.0\%(图6c), 但值得注意的是, 秋季的响应模 式都是对于生长季终止点而言的. 这表明秋季GPP由 于EOS延迟主要表现为增加, 这可能跟更长的光合作 用期有关, 而秋季ET由于EOS延长带来更长的蒸腾作 用期以及更低的地表蒸发, 从而表现出不确定性. 但 即使ET增加, 增加的强度也小于GPP增加的强度.

进一步分析季节性WUE对物候变化的响应模式 随气候梯度的空间变异. 按像元的多年平均气候值排 序, 从低值起每 200 个像元为一组, 进行WUE响应模式 统计, 结果如图7所示, 横轴表征每组的气候均值, 纵轴
表示各模式占比. 结果表明, 在春季, 各温度区间均由 模式VI主导, 模式 V 和模式 IV 次之, 其他模式占比较 小; 各模式随温度梯度的变化均不明显, 总体而言, 模 式IV 随着温度升高整体呈现一定下降趋势. 沿降水率 梯度, 响应模式变化明显: 总体而言, 低降水区, 模 式IV 占主导地位, 而在高降水区, 模式VI占主导地位. 当降水率小于 $0.22 \mathrm{~mm} \mathrm{~h}^{-1}$, 模式VI随降水增加而增加, 模式IV 随降水的增加而减少, 当降水率大于 $0.22 \mathrm{~mm}$ $\mathrm{h}^{-1}$, 模式IV 维持在低值水平(约为 $3 \%$ ), 而模式VI维持 在高值水平(约为 $67 \%$ ), 结合这两者变化可以发现, 随 着降水的增加, 存在春季模式IV 向模式VI转移的现象. 以上结果表明, 即使生长季提前, 如果春季降水不足, 植被光合作用受到水分限制, 地表和冠层截留蒸发以 及叶片蒸腾作用减弱, GPP和ET仍会下降. 对于辐射梯 度变化结果而言, 低辐射区, 模式VI占主导地位, 中辐 射地区, 模式IV 占主导地位, 高辐射地区无主导模式. 从低辐射地区到中辐射地区, 可以看出模式VI在逐渐 向模式IV转移. 在夏季, 模式 I 和 II 大量存在于各个温 度区间, 其余模式所占比重很小, 但随着温度梯度模 式 II 在逐渐减少, 从 $54.0 \%$ 下降到 $15.5 \%$, 模式 IV 和 V 逐 渐增大, 从 $5 \%$ 下降到 $33 \%$, 模式 II 随着温度的增加逐渐 向模式 $I V$ 和 $V$ 转移. 随降水率和辐射梯度, 响应模式变 化规律均不明显, 总体而言, 模式 I 和 II 随降水率和辐 射梯度变化虽有不同程度波动但均大量存在各区间中. 在秋季, 各温度区间均由模式 I 主导, 其次是模式 II, 其余模式占比不高, 但模式 I 变化规律不明显. 总体而 言, 模式 II 随着温度升高呈现轻微上升趋势, 模式IV 虽 然占比较小且在低温地区接近为 0 , 但随着温度升高表 现出增加. 随降水率梯度, 响应模式变化明显: 低降水 区, 模式 I 和 II 所占比重相当, 而高降水区, 模式 I 占 主导地位, 最大值达 $89 \%$. 与降水率梯度结果类似, WUE响应模式随辐射梯度表现低辐射区模式 I 和 II 比 重高且所占比重相当, 而高辐射区模式 I 成为主导模 式. 这两种变化情况表明, 当秋季EOS延迟时, 降水和 辐射的增加通过为森林生态系统提供水分和能量能增 加秋季GPP、ET以及WUE.

\section{5 讨论}

\section{1 物候变化对季节WUE的影响}

本研究基于季节的WUE时空动态变化结果显示, 


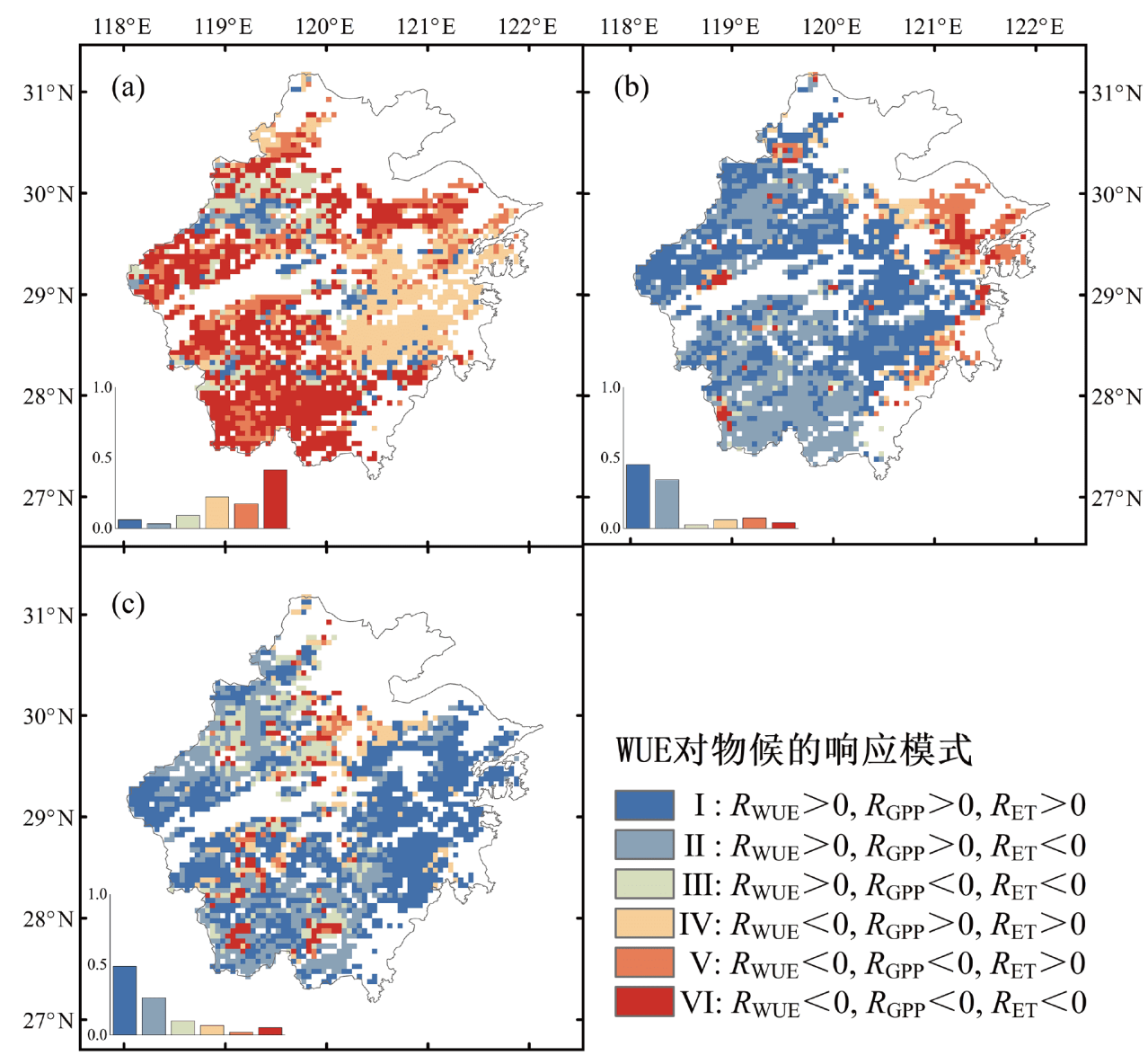

图 6 季节性水分利用效率(WUE)对物候期(SOS/EOS)响应模式的空间分布

$R_{\mathrm{WUE}} 、 R_{\mathrm{GPP}} 、 R_{\mathrm{ET}}$ 分别表示在控制气候因子(温度、降水、辐射)后WUE、GPP、ET与物候期的偏相关系数. (a) (c)分别对应春季、夏季、秋 季. 白色区域为无效像元

研究区春夏两季的WUE整体表现为逐年上升的态势, 而秋季的WUE大范围地表现为逐年下降的态势，并且 下降幅度明显大于春夏两季上升幅度总和。Sun等 (2018)研究了中国过去30年WUE年际变化趋势空间分 布，结果显示浙江地区年际WUE在逐年降低(Sun等, 2018). 本研究支持这一观点，并进一步说明其年际趋 势下降的主要原因是该区域秋季WUE在大面积大幅 度下降.

对于亚热带森林生态系统, 春季WUE与SOS主要 表现为负相关，且春季大量存在的模式 VI和 $V$ 表明, $\operatorname{SOS}$ 提前幅度越大, 植物越早开始发芽或者复苏, 植被 越早开始光合作用, GPP主要表现为增加的趋势(Luyssaert等，2007), 而由于春季较大的叶面积在增加植物 的蒸腾量的同时也通过降低地表温度以及太阳辐射减 少地表蒸发, 从而春季ET变化表现出不确定性(Beer
等，2009; von Arx等，2012; Shen等，2015). 但模 式VI和 $\mathrm{V}$ 中都大于 0 的 $R_{\mathrm{WUE}}$ 进一步表明, 即使 $\mathrm{ET}$ 总量 在增加, 增加幅度仍小于 GPP增加幅度, 从而春季 WUE随SOS提前而增加. 对于夏季而言, WUE与SOS 主要表现为正相关, 这可能与夏季GPP对SOS的响应 有关: 一方面, SOS提前, 可能会过早耗水, 造成夏季 土壤水分对植被生理过程的限制(Kljun等，2006; Hu 等, 2010), 降低GPP, 即SOS提前, GPP下降; 另一方面, 从碳经济和生态对策角度, 若 SOS较正常年份相比开 始得晚, 为保证正常生长, 植被在生长季会有更强的 固碳表现, 即SOS延后, GPP增加. 从夏季WUE对SOS 的响应模式来看, 大量存在的模式 I 和 II 进一步支持 了以上假设. 对于秋季而言, WUE与EOS同样主要表 现为正相关, 因为EOS越迟, 为生态系统的光合作用 争取到了额外的光合作用时间, 增加了秋季GPP; 而秋 

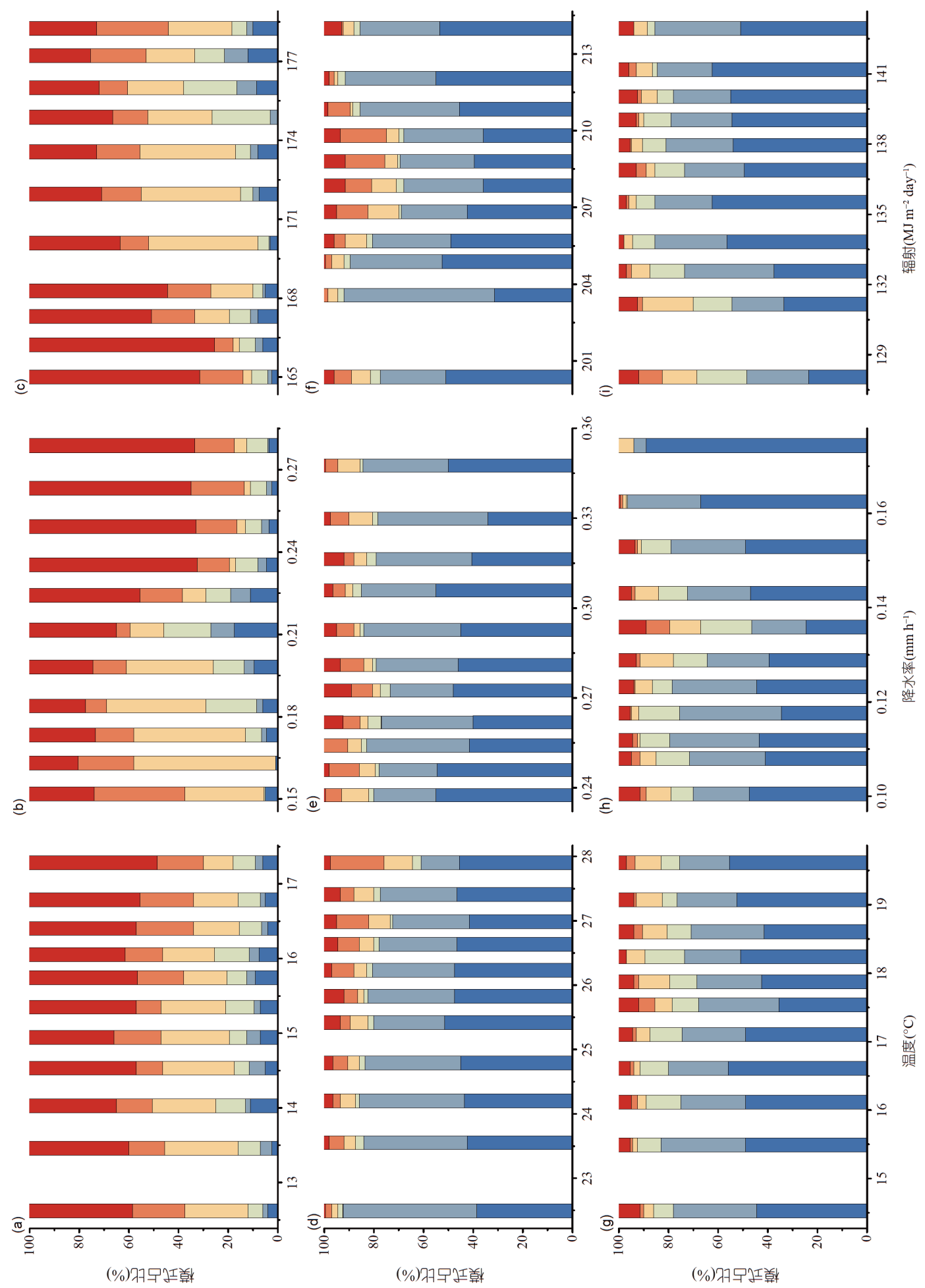
变

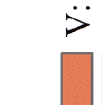


季温度和土壤水分往往较低，不会显著提高土壤或者 冠层蒸发, 进而秋季ET变化不大或减小.

\section{2 不同梯度下WUE对物候敏感性的变化}

春季WUE与SOS的敏感性与辐射表现出正相关, 即在辐射越高的区域，SOS提前对春季WUE的促进作 用越弱. 高辐射地区模式 VI 向模式III转移(模式III WUE与SOS的正相关性抵消了一部分负相关性), 表明 在高辐射地区，虽然GPP、ET与SOS仍然表现为负相 关, 但是由于春季植物叶面积相对较小, 穿透冠层到 达地面的辐射较多, 促进了土壤蒸发进而增加ET, 导 致春季WUE增加幅度的减弱.

夏季WUE与SOS的敏感性和空间降水和温度均 显著相关. 夏季WUE对SOS响应的潜在原因为与SOS 相关的水分限制和生态对策, 而研究区SOS主要表现 为延后, 因此夏季GPP对生长季延迟的补偿作用可能 是造成WUE与SOS正相关的主要原因. 值得注意的是, 通常我们认为提前的SOS会过早消耗土壤水分才会导 致夏季GPP受限, 但在SOS表现为延后的本研究区, 夏 季高温造成的水分胁迫可能成为了夏季GPP的主要限 制因子, 因此, 在降水少、温度高的地区，植物为保证 水分减少蒸滕作用, 气孔闭合, 光合作用受限, 从而夏 季GPP对生长季延后的补偿作用会因水分胁迫而受到 限制，因此WUE与SOS的正相关性较弱; 而在降水 多、温度低的地区，一方面由于降水的增加，植物叶 片气孔由闭合逐渐变成微张状态，此时途经气孔的碳 交换大于水交换，另一方面，低温地区生长季已经较 短, 生长季开始期一旦延后, 植物为保证自身生长需 求，只能通过提高夏季GPP来获取生长发育所需养分 (这与低温地区模式 I 和 II 之和大于高温地区这一结 果相吻合)，这两方面都会导致夏季GPP的补偿作用表 现更为明显, 因此WUE与SOS正相关性较强. 夏季 WUE与SOS的敏感性随降水增加变化趋势较小，且 WUE与SOS的响应模式随降水梯度变化特征不明显, 但夏季随温度梯度减少的模式 II $\left(R_{\mathrm{GPP}}>0\right)$ 以及增加的 模式 $\mathrm{V}\left(R_{\mathrm{GPP}}<0\right)$ 也进一步说明夏季 $\mathrm{GPP}$ 与 $\mathrm{SOS}$ 的正相 关关系随温度增加而减弱. 因此, 典型亚热带森林生态 系统WUE对物候敏感性不仅随不同气候梯度表现出 不同的变化特征，还跟研究区当地的生境条件密切相 关. 这一结果对WUE预测及碳收支评估相关研究有一 定的指示意义, 即在考虑未来情景气候变化(例如气候
变暖或者变湿)的同时, 也要考虑当地的植物生境条件 (例如干旱或者湿润).

对于秋季而言，其WUE对EOS的敏感性在空间上 随降水和辐射显著变化. 具体而言, 沿降水梯度, EOS 延迟对秋季WUE的促进作用逐渐增强. 经过春夏两季 的植物吸收、蒸腾和地表蒸发，秋季土壤水分含量往 往较低. EOS延迟意味着更长的光合作用时间, 更为丰 富的降水可以增加土壤湿度, 从而增强植物生理活 性、支持秋季延长的光合作用，因此提高秋季WUE. 同时, 秋季沿降水梯度整体表现为模式 I 增加, 也验证 了这一观点. 沿辐射梯度表现出类似的现象, 且高辐射 地区, 模式 I 、 II 占比相对较高, 说明了在EOS延迟的 森林生态系统, 秋季的辐射为秋季植物光合作用提供 能量(Jin等, 2017a), 对其GPP和WUE都具有促进作用.

\section{6 结论}

本文研究了不同季节生态系统尺度WUE与物候 的年际趋势及其相关关系, 识别了WUE对物候的响应 模式, 并进一步结合不同气候梯度下季节性WUE对物 候的响应模式，分析了不同气候梯度下亚热带常绿落 叶阔叶混交林季节性WUE对物候响应差异的潜在原 因. 本研究发现, 亚热带常绿落叶阔叶混交林季节性 WUE整体上与物候密切相关. 同时, 不同季节WUE对 物候敏感性对应不同的主导气候因子并随其呈现不同 的变化特征. 春季WUE对 SOS的敏感性随辐射梯度显 著增强，夏季低温高湿地区WUE对SOS的敏感性强于 高温低湿地区，秋季WUE对EOS的敏感性随降水率和 辐射梯度变化显著, 高辐射高降水地区WUE对EOS的 敏感性较强. 这些结果表明, 气候变化会改变亚热带常 绿落叶阔叶混交林WUE对物候的敏感性, 并且在未来 生态系统过程模型的参数设定以及碳收支评估时，有 必要考虑不同情景下的气候变化以及当地生境条件下 亚热带常绿落叶阔叶混交林WUE的生物调控机制.

\section{参考文献}

Beer C, Ciais P, Reichstein M, Baldocchi D, Law B E, Papale D, Soussana J F, Ammann C, Buchmann N, Frank D, Gianelle D, Janssens I A, Knohl A, Köstner B, Moors E, Roupsard O, Verbeeck H, Vesala T, Williams C A, Wohlfahrt G. 2009. Temporal and among-site variability of inherent water use efficiency at the 
ecosystem level. Glob Biogeochem Cycle, 23: GB2018

Chen Y Y, Yang K, He J, Qin J, Shi J C, Du J Y, He Q. 2011. Improving

land surface temperature modeling for dry land of China. J Geophys Res, 116: D20104

Dai J H, Xu Y J, Wang H J, Alatalo J, Tao Z X, Ge Q S. 2017. Variations in the temperature sensitivity of spring leaf phenology from 1978 to 2014 in Mudanjiang, China. Int J Biometeorol, 63: 569-577

Fu Y S H, Campioli M, Vitasse Y, De Boeck H J, Van den Berge J, AbdElgawad H, Asard H, Piao S, Deckmyn G, Janssens I A. 2014. Variation in leaf flushing date influences autumnal senescence and next year's flushing date in two temperate tree species. Proc Natl Acad Sci USA, 111: 7355-7360

Ge Q S, Dai J H, Cui H J, Wang H J. 2016. Spatiotemporal variability in start and end of growing season in china related to climate variability. Remote Sens, 8: 433

Guerrieri R, Lepine L, Asbjornsen H, Xiao J, Ollinger S V. 2016. Evapotranspiration and water use efficiency in relation to climate and canopy nitrogen in U.S. forests. J Geophys Res-Biogeosci, 121: $2610-2629$

Huang G, Li Y, Mu X H, Zhao H M, Cao Y F. 2017. Water-use efficiency in response to simulated increasing precipitation in a temperate desert ecosystem of Xinjiang, China. J Arid Land, 9: 823836

Huang M T, Piao S L, Sun Y, Philippe C, Cheng L, Mao J F, Ben P, Shi X Y, Zeng Z Z, Wang Y P. 2015. Change in terrestrial ecosystem water-use efficiency over the last three decades. Glob Change Biol, 21: $2366-2378$

Hu J, Moore D J P, Burns S P, Monson R K. 2010. Longer growing seasons lead to less carbon sequestration by a subalpine forest. Glob Change Biol, 16: 771-783

Jin J X, Wang Y, Zhang Z F, Magliulo V, Jiang H, Cheng M. 2017a. Phenology plays an important role in the regulation of terrestrial ecosystem water-use efficiency in the northern hemisphere. Remote Sens, 9: 664

Jin J X, Zhan W F, Wang Y, Gu B, Wang W, Jiang H. 2017b. Water use efficiency in response to interannual variations in flux-based photosynthetic onset in temperate deciduous broadleaf forests. Ecol Indic, 79: 122-127

Keenan T F, Hollinger D Y, Bohrer G, Dragoni D, Munger J W, Schmid H P, Richardson A D. 2013. Increase in forest water-use efficiency as atmospheric carbon dioxide concentrations rise. Nature, 499: 324-327

Keenan T F, Gray J, Friedl M A, Toomey M, Bohrer G, Hollinger D Y, Munger J W, O'Keefe J, Schmid H P, Wing I S, Yang B, Richardson A D. 2014. Net carbon uptake has increased through warming- induced changes in temperate forest phenology. Nat Clim Change, 4: 598-604

Keenan T F, Richardson A D. 2015. The timing of autumn senescence is affected by the timing of spring phenology: Implications for predictive models. Glob Change Biol, 21: 2634-2641

Kljun N, Black T A, Griffis T J, Barr A G, Gaumont-Guay D, Morgenstern K, McCaughey J H, Nesic Z. 2006. Response of net ecosystem productivity of three boreal forest stands to drought. Ecosystems, 9: 1128-1144

Law B E, Falge E, Gu L, Baldocchi D D, Bakwin P, Berbigier P, Davis K, Dolman A J, Falk M, Fuentes J D, Goldstein A, Granier A, Grelle A, Hollinger D, Janssens I A, Jarvis P, Jensen N O, Katul G, Mahli Y, Matteucci G, Meyers T, Monson R, Munger W, Oechel W, Olson R, Pilegaard K, Paw U K T, Thorgeirsson H, Valentini R, Verma S, Vesala T, Wilson K, Wofsy S. 2002. Environmental controls over carbon dioxide and water vapor exchange of terrestrial vegetation. Agric For Meteorol, 113: 97-120

Le Houerou H N. 1984. Rain use efficiency: A unifying concept in aridland ecology. J Arid Environ, 7: 213-247

Li Y, Li C H, Xie J B, Liu Y, Liu X C, Wang Y G. 2019. Accumulation of organic carbon and its association with macro-aggregates during 100 years of oasis formation. Catena, 172: 770-780

Liu R, Li Y, Wang Y G, Ma J, Cieraad E. 2019. Variation of water use efficiency across seasons and years: Different role of herbaceous plants in desert ecosystem. Sci Total Environ, 647: 827-835

Liu R, Wang Y G, Li C H, Ma J, Li Y. 2018. Partitioning water source and sinking process of a groundwater-dependent desert plant community. Plant Soil, 430: 73-85

Liu Q, FuY S H, Zhu Z C, Liu Y W, Liu Z, Huang M T, Janssens I A, Piao S L. 2016. Delayed autumn phenology in the northern hemisphere is related to change in both climate and spring phenology. Glob Change Biol, 22: 3702-3711

Luyssaert S, Janssens I A, Sulkava M, Papale D, Dolman A J, Reichstein M, Hollmén J, Martin J G, Suni T, Vesala T, Loustau D, Law B E, Moors E J. 2007. Photosynthesis drives anomalies in net carbon-exchange of pine forests at different latitudes. Glob Change Biol, 13: 2110-2127

McPherson R A. 2007. A review of vegetation-Atmosphere interactions and their influences on mesoscale phenomena. Prog Phys Geogr, 31: 261-285

Morisette J T, Richardson A D, Knapp A K, Fisher J I, Graham E A, Abatzoglou J, Wilson B E, Breshears D D, Henebry G M, Hanes J M, Liang L. 2009. Tracking the rhythm of the seasons in the face of global change: Phenological research in the 21st century. Front Ecol Environ, 7: 253-260

Mu Q, Zhao M, Running S W. 2011. Improvements to a modis global 
terrestrial evapotranspiration algorithm. Remote Sens Environ, 115: 1781-1800

Piao S L, Tan J G, Chen A P, Fu Y S H, Philippe C, Liu Q, Janssens I A, Sara V, Zeng Z Z, Jeong S J L Y, Myneni R B, Peng S S, Shen M G, Peñuelas J. 2015. Leaf onset in the northern hemisphere triggered by daytime temperature. Nat Commun, 6: 6911

Ponton S, Flanagan L B, Alstad K P, Johnson B G, Morgenstern K, Kljun N, Black T A, Barr A G. 2010. Comparison of ecosystem water-use efficiency among Douglas-fir forest, aspen forest and grassland using eddy covariance and carbon isotope techniques. Glob Change Biol, 12: 294-310

Reed B C, Schwartz M D, Xiao X. 2009. Phenology of Ecosystem Processes. New York: Springer

Reichstein M, Ciais P, Papale D, Valentini R, Running S, Viovy N, Cramer W, Granier A, Ogée J, Allard V, Aubinet M, Bernhofer C, Buchmann N, Carrara A, Grünwald T, Heimann M, Heinesch B, Knohl A, Kutsch W, Loustau D, Manca G, Matteucci G, Miglietta F, Ourcival J M, Pilegaard K, Pumpanen J, Rambal S, Schaphoff S, Seufert G, Soussana J F, Sanz M J, Vesala T, Zhao M. 2010. Reduction of ecosystem productivity and respiration during the european summer 2003 climate anomaly: A joint flux tower, remote sensing and modelling analysis. Glob Change Biol, 13: 634-651

Richardson A D, Andy Black T, Ciais P, Delbart N, Friedl M A, Gobron N, Hollinger D Y, Kutsch W L, Longdoz B, Luyssaert S, Migliavacca M, Montagnani L, William Munger J, Moors E, Piao S, Rebmann C, Reichstein M, Saigusa N, Tomelleri E, Vargas R, Varlagin A. 2010. Influence of spring and autumn phenological transitions on forest ecosystem productivity. Phil Trans R Soc B, 365: 3227-3246

Richardson A D, Keenan T F, Migliavacca M, Ryu Y, Sonnentag O, Toomey M. 2013. Climate change, phenology, and phenological control of vegetation feedbacks to the climate system. Agric For Meteorol, 169: 156-173

Running S W, Nemani R R, Heinsch F A, Zhao M, Reeves M, Hashimoto H. 2004. A continuous satellite-derived measure of global terrestrial primary production. Bioscience, 54: 547-560

Shen M G, Piao S L, Jeong S J, Zhou L M, Zeng Z Z, Ciais P, Chen D, Huang M T. 2015. Evaporative cooling over the tibetan plateau induced by vegetation growth. Proc Natl Acad Sci USA, 112: 92999304

Sun S B, Song Z L, Wu Y T, Wu X C, Wang T J, Du W L, Che T, Huang C L, Zhang X J, Ping B, Lin X F, Li P, Yang Y X, Chen B Z. 2018. Spatio-temporal variations in water use efficiency and its drivers in China over the last three decades. Ecol Indic, 94: 292-304
Sun Y, Piao S L, Huang M T, Ciais P, Zeng Z Z, Cheng L, Li X R, Zhang X P, Mao J F, Peng S S. 2016. Global patterns and climate drivers of water-use efficiency in terrestrial ecosystems deduced from satellite-based datasets and carbon cycle models. Glob Ecol Biogeogr, 25: 311-323

Tang X G, Li H P, Desai A R, Nagy Z, Luo J H, Kolb T E, Olioso A, Xu X B, Yao L, Kutsch W, Pilegaard K, Köstner B, Ammann C. 2015. How is water-use efficiency of terrestrial ecosystems distributed and changing on earth? Sci Rep, 4: 7483

von Arx G, Dobbertin M, Rebetez M. 2012. Spatio-temporal effects of forest canopy on understory microclimate in a long-term experiment in switzerland. Agric For Meteorol, 166-167: 144-155

Wang H J, Dai J H, Zheng J, Ge Q S. 2015. Temperature sensitivity of plant phenology in temperate and subtropical regions of China from 1850 to 2009. Int J Climatol, 35: 913-922

Wang H J, Zhong S Y, Tao Z X, Dai J H, Ge Q S. 2017. Changes in flowering phenology of woody plants from 1963 to 2014 in North China. Int J Biometeorol, doi: 10.1007/s00484-017-1377-2

Wang H J, Dai J H, Rutishauser T, Gonsamo A, Wu C Y, Ge Q S. 2018. Trends and variability in temperature sensitivity of lilac flowering phenology. J Geophys Res-Biogeosci, 123: 807-817

Wolf S, Keenan T F, Fisher J B, Baldocchi D D, Desai A R, Richardson A D, Scott R L, Law B E, Litvak M E, Brunsell N A, Peters W, van der Laan-Luijkx I T. 2016. Warm spring reduced carbon cycle impact of the 2012 US summer drought. Proc Natl Acad Sci USA, 113: 5880-5885

Wu C Y, Chen J M, Gonsamo A, Price D T, Black T A, Kurz W A. 2012. Interannual variability of net carbon exchange is related to the lag between the end-dates of net carbon uptake and photosynthesis: Evidence from long records at two contrasting forest stands. Agric For Meteorol, 164: 29-38

Wu C Y, Chen J M, Black T A, Price D T, Kurz W A, Desai A R. 2013. Interannual variability of net ecosystem productivity in forests is explained by carbon flux phenology in autumn. Glob Ecol Biogeogr, 22: 994-1006

Yang Y T, Guan H D, Shang S H, Long D, Craig T S. 2014. Toward the use of the modis et product to estimate terrestrial gpp for nonforest ecosystems. IEEE Geosci Remote Sens Lett, 11: 1624-1628

Zhao M S, Heinsch F A, Nemani R R, Running S W. 2005. Improvements of the modis terrestrial gross and net primary production global data set. Remote Sens Environ, 95: 164-176

Zhou S, Yu B F, Huang Y F, Wang G Q. 2014. The effect of vapor pressure deficit on water use efficiency at the subdaily time scale. Geophys Res Lett, 41: 5005-5013

(责任编委: 李彦) 